\title{
De la tutoría presencial a la virtual: la evolución del proceso de tutorización
}

From on-site tutoring to virtual one: evolution of tutoring process

Yolanda Giner Manso'

María José Muriel de los Reyes

F. Javier Toledano Redondo

Universidad de Cádiz, España

\section{Resumen}

La tutoría es uno de los recursos metodológicos que, sin duda, más ha evolucionado tras la implantación del Espacio Europeo de Educación Superior (EEES). La concepción de la tutoría meramente como una obligación legal de atender las dudas de los estudiantes ha dado paso al uso de la tutoría como una de las metodologías más útiles para desarrollar determinadas capacidades y competencias, adicionalmente a su contribución al aprendizaje de la materia en la que se inserta.

El presente trabajo analiza, desde la perspectiva de experiencias de innovación docentes desarrolladas, la evolución de la tutoría tradicional hasta el uso que actualmente se puede hacer de ella como foro interactivo entre iguales, y propone una clasificación de la misma.

Finalmente, se expone un nuevo avance en el papel de la tutoría apoyada en las nuevas tecnologías como medio idóneo para el contraste de opiniones, el fomento del espíritu crítico y la construcción del pensamiento autónomo del estudiante

Palabras clave. Educación superior, tutoría presencial, tutoría virtual, nuevas tecnologías, experiencias de innovación.

\begin{abstract}
Tutoring is one of the methodological resources which has undoubtedly experienced a deepest evolution since the implantation of the European Higher Education Area (EHEA). Tutorial conception as a merely legal obligation to address students' doubts has given rise to the use of tutorials as one of the most useful methods to develop certain skills and competences, in addition to its contribution to the learning of the subject in which they are inserted.
\end{abstract}

${ }^{1}$ Los autores aparecen por estricto orden alfabético. 
This paper analyzes, from the perspective of developed innovative teaching experiences, the evolution of traditional mentoring until the current use that can be made of them as an interactive forum of equals, and, moreover, suggesting a classification.

Finally, this paper shows a new breakthrough in the role of tutoring supported on new technologies as a suitable means of contrasting opinions, encouraging critical thinking and building student's autonomous thought.

Key words: Higher Education, on-site tutoring, virtual tutoring, new technologies, innovation experiences.

\section{Introducción}

El debate existente en torno a la calidad de la enseñanza universitaria ha puesto en primer plano la función tutorial como una actividad de apoyo al proceso educativo, de mejora del proceso de aprendizaje (Álvarez, 2005-2006), como una labor de asesoramiento personalizado del aprendizaje. Si hasta ahora la acción tutorial ha estado sujeta a la iniciativa de cada profesor, en el EEES, por exigencia implícita, la tutorización pasa a tener una consideración especial como metodología docente. La función docente no se puede identificar solo con la enseñanza sino que ha de abarcar la orientación y tutoría de los alumnos (Sola y Moreno, 2005; Castaño et al., 2012).

La tutoría en el contexto del EEES debe ser una tutoría integral, donde se oriente al alumno no sólo en el aprendizaje de una determinada materia sino, además, y sin obviar el contexto de cada asignatura, se oriente al estudiante en competencias profesionales y personales. Coincidimos con las reflexiones de Castaño et al. (2012) al entender que de nada sirve un estudiante con un excelente bagaje académico si no da un paso más y añade a sus competencias de conocimientos y capacidades un desarrollo integral que le permita aprender de forma continua.

La actual visión y paradigma para la formación de los estudiantes basados en el aprendizaje continuo, a lo largo de toda la vida, la orientación prioritaria hacia el aprendizaje autodirigido y el diseño de nuevas modalidades educativas en las cuales el alumno sea el actor central en el proceso formativo hace que el profesor deje de ser la única y principal fuente de información para el alumno, para convertirse en colaborador, guía y facilitador de estrategias de aprendizaje. El principal papel del profesor se centra por tanto más en motivar y posibilitar el aprendizaje del alumno que en transmitir conocimientos (Álvarez, 2008).

Ya en 1996 García y Gálvez mostraban su preocupación por el desarrollo de experiencias válidas de tutorías en el contexto universitario. Aspectos como la masificación, la premura de espacios y tiempos, el sentimiento de anonimato que viven los alumnos y la percepción por parte de los profesores y alumnos de la imposibilidad de conseguir la personalización educativa motivan la falta de iniciativas.

Adicionalmente, como exponen Lobato et al. (2005), los profesores universitarios llegan al ejercicio de la profesión sin formación en materias pedagógicas, psicológicas y sociológicas y esta formación la adquieren en la práctica docente y a partir de sus propias experiencias. Igualmente, cada profesor desempeña su labor tutorial según sus experiencias, motivación por enseñar, expectativas, confianza, optimismo, 
preocupaciones sobre la enseñanza, su percepción del papel y responsabilidad como tutor.

El presente trabajo constituye una reflexión crítica sobre la evolución de la tutoría universitaria en el contexto de varias asignaturas de finanzas impartidas en la Facultad de Ciencias Económicas y Empresariales de la Universidad de Cádiz, si bien la naturaleza de las experiencias planteadas es, en gran medida, independiente de la asignatura en la que se inserta y, por tanto, es aplicable a otros contextos. Su estructura es la siguiente: tras analizar en el segundo apartado la evolución de la acción tutorial, en el tercer epígrafe se recoge una propuesta de clasificación de la misma acorde con las experiencias desarrolladas como tutores por los autores, recogidas en el apartado cuatro. Para finalizar se sintetizan las principales conclusiones alcanzadas.

\section{Evolución de la acción tutorial}

La actividad docente universitaria ha venido evolucionando a lo largo de la historia, pasando desde la pura disertación magistral hasta llegar a modelos de participación en un proceso de enseñanza-aprendizaje virtual. Las nuevas exigencias implican una modificación en la función docente para pasar de una función centrada en la enseñanza al aprendizaje, mientras que el alumno, por su parte, pasa de ser un elemento pasivo a tener un papel preponderante en el proceso de aprendizaje, activo y protagonista del proceso de adquisición de conocimientos, capacidades y competencias. Si bien el profesorado universitario ha realizado un gran esfuerzo en la adaptación de metodologías y materiales docentes a las exigencias de las nuevas titulaciones de Grado (Capelo et al., 2010), el desarrollo de la acción tutorial no ha seguido el mismo ritmo.

Como señala Sancho (2002), desde una visión amplia, la tutoría universitaria habría que entenderla como el conjunto de todas las actividades, actitudes, procesos, intercambios personales y profesionales que caracterizan la relación entre el docente y los estudiantes.

En este nuevo marco, la tutoría ha de considerarse responsabilidad del profesor con los alumnos, contribuyendo a su formación no meramente académica, sino integral: profesional, social y personal.

Según Gallego (1997), la tutoría se define como un proceso orientador en el que el profesor-tutor y el alumno se encuentran en un espacio común para, de mutuo acuerdo, con un marco teórico referencial y una planificación previa, el primero ayude al segundo en aspectos académicos y/o profesionales y juntos puedan establecer un proyecto de trabajo que conduzca a la posibilidad de que el estudiante pueda diseñar y desarrollar su plan de carrera. De tal definición se desprende que la tutoría no siempre es igual, no se puede estandarizar (Barrón, 2009).

El tutor o asesor académico, por tanto, es la persona que ejerce la función de guiar, orientar e informar al estudiante en su formación intelectual, académica y profesional (García et al., 2005). 
En sus orígenes, la tutoría tiene una vocación de atención individualizada por alguien que se encuentra más capacitado que el alumno o aprendiz para dirigir y apoyar su aprendizaje; no obstante, existen diversas formar de concebir esa labor dando lugar a distintos modelos de actuación.

EI EEES con la implantación del Sistema de Créditos Europeos (ECTS) centra la atención en el aprendizaje y en el trabajo del estudiante universitario, lo que supone un nuevo papel del tutor como guía de los procesos de adquisición y maduración de los aprendizajes de los estudiantes.

No obstante, tal y como afirman Álvarez y González (2008), existen otros muchos motivos, de naturaleza diversa, que han conducido a la puesta en marcha de experiencias orientadoras encaminadas a paliar sus efectos, tales como la competencia entre universidades por captar alumnado, los altos índices de fracaso y abandono en los primeros cursos, las dificultades para adaptarse a la dinámica y exigencias del sistema de Educación Superior, la escasa información y orientación con la que llega el alumnado a la Universidad, las dificultades para abordar las tareas académicas, para recorrer los itinerarios formativos o la desinformación sobre salidas profesionales.

\section{Modalidades de la acción tutorial}

La tutoría se manifiesta de diversas maneras según los niveles educativos, los ámbitos, los recursos empleados, los agentes que intervienen y los contenidos que trata.

En el presente trabajo, que recogen las experiencias de un grupo de profesores del Área de Economía Financiera de la Universidad de Cádiz, se parte de una definición de tutoría académica universitaria para, basándonos en la misma, clasificar y exponer las distintas modalidades desarrolladas durante el ejercicio de la profesión como docentes.

Según Sogues et al. (2007), la tutoría académica universitaria se entiende como una concreción del proceso de orientación, una estrategia de carácter formativo, orientador e integral desarrollado por docentes universitarios con la finalidad de orientar al alumno en su proceso formativo y madurativo.

No obstante, compartimos con Álvarez (2008) la opinión de que, en el contexto del EEES, se trata de hacer más explícita y sistemática la función que cada profesor desarrolla de forma espontánea como guía y facilitador del proceso de aprendizaje de sus alumnos, tanto a nivel individual como grupal.

Tras la revisión de la literatura previa sobre modalidades de tutoría, recogida en Álvarez y González (2008), siguiendo la clasificación integradora que proponen y basándonos en la experiencia acumulada por los autores del presente trabajo, se definen brevemente a continuación distintos tipos de acción tutorial que en un epígrafe posterior serán argumentadas con casos reales. 


\section{Tutoría académica: presencial y virtual}

El profesor es tutor de un grupo más o menos numeroso de alumnos a los que les imparte docencia de una asignatura, habitualmente en el aula aunque también tiene cabida la docencia semipresencial. El número de alumnos depende del curso al que pertenezca la asignatura y del carácter de la misma (obligatoria u optativa). Normalmente suele ser tutoría individualizada (tutoría individual) o en grupo muy reducido (tutoría grupal).

El caso de las tutorías grupales, especialmente en el ámbito del EEES, puede constituir una actividad metodológica más, y su desarrollo está incluido en la planificación docente, por lo que el alumno debe conocer el contenido de dicha tutoría y su asistencia es generalmente obligatoria, al igual que el resto de sesiones presenciales de la materia.

Las tutoría individuales, no obstante, suelen tener otra concepción, dado que a no ser que el profesor cite expresamente al alumno, es el estudiante quien accede libremente según lo estime conveniente. La finalidad es atender al alumno sobre todo en la comprensión de la materia (teoría y práctica), revisión de exámenes o elaboración de trabajos propuestos.

El tiempo de dedicación es de seis horas semanales de permanencia en el propio despacho; es lo que se denomina tutoría legal o funcional contemplada por la normativa legal nacional previa a la implantación del EEES, que contempla que todo profesor universitario con dedicación exclusiva ha de atender seis horas semanales de tutoría. Para algunos profesores, la tutoría se convierte así en una tarea de obligado cumplimiento en la que, a petición del alumno, se atienden dudas o resuelven consultas de notas o exámenes (Álvarez y González, 2008).

Si se utilizan los recursos que proporcionan las tecnologías de la información y comunicación para entablar la relación entre el profesor y el alumno (correo electrónico, chat, foro, diálogo,...) entonces se denomina tutoría virtual.

\section{Tutoría entre iguales}

Consiste en la labor de ayuda desempeñada por alumnos, generalmente de una misma titulación, si bien existen otras modalidades que se desarrollan, por ejemplo, en programas de acompañamiento de alumnos extranjeros que no tienen que pertenecer al mismo ámbito de estudios del alumno tutor y donde el nexo de unión puede ser simplemente el conocimiento del idioma común.

La tutorización puede desarrollarse entre alumnos que cursan una misma asignatura, en cuyo caso se trataría de una tutoría académica entre iguales, o entre alumnos de distintos cursos con el fin de ayudar en la adaptación, integración y aprendizaje a los alumnos de los primeros cursos. Se trata, pues, de que los alumnos experimentados y conocedores del medio universitario por experiencia personal apoyen y orienten a aquellos alumnos nuevos y sin experiencia por ser recién llegados a la universidad o estar en los primeros cursos. No obstante, en ambos casos, puede existir una supervisión por parte del profesor que guíe y oriente las sesiones tutoriales. 
La principal ventaja de esta modalidad de tutoría es que reduce la distancia y refuerza la empatía entre los interlocutores.

Generalmente, el actuar como alumno tutor tiene un carácter voluntario, por lo que será necesario utilizar principios de administración de voluntarios, que incluyan la atención a las necesidades e inquietudes de todas las partes involucradas.

\section{Propuesta de clasificación de la acción tutorial a través de experiencias de innovación docente}

La evolución del sistema de tutoría implantado en diversas asignaturas del Área de Economía Financiera de las titulaciones de la rama de Empresa de la Universidad de Cádiz abarca, aproximadamente, los últimos diez años, desde la primera evaluación de la tutoría, y nuestra preocupación por su escaso uso y las reticencias del alumnado a su empleo más allá, en el mejor de los casos, de resolver algunas dudas puntuales relacionadas con la materia.

La siguiente tabla recoge, siguiendo la clasificación inicial, todas las experiencias de tutoría desarrolladas, hasta la fecha. Para su ordenación los criterios tenidos en cuenta han sido la participación del profesorado en el proceso de tutorización (tutela efectiva, supervisión del proceso o no supervisión en la tutoría entre iguales) y el nivel de presencialidad en las tutorías (presencial, semipresencial o virtual).

\begin{tabular}{|c|c|c|c|c|}
\hline $\begin{array}{l}\text { Participación } \\
\text { del equipo } \\
\text { docente }\end{array}$ & $\begin{array}{l}\text { Nivel de } \\
\text { presencia- } \\
\text { lidad }\end{array}$ & Tipología & Contenido & Objetivo principal \\
\hline \multirow{4}{*}{$\begin{array}{l}\text { Tutoría } \\
\text { académica } \\
\text { con el equipo } \\
\text { docente }\end{array}$} & \multirow{3}{*}{$\begin{array}{l}\text { Presen- } \\
\text { ciales }\end{array}$} & $\begin{array}{l}\text { Individual } \\
\text { (tutoría legal) }\end{array}$ & $\begin{array}{l}\text { El estudiante resuelve } \\
\text { dudas con el profesor }\end{array}$ & $\begin{array}{l}\text { Adquisición de } \\
\text { conocimientos y } \\
\text { competencias específicas. } \\
\text { Facilitar la creación de un } \\
\text { espacio en el que el } \\
\text { alumno pueda plantear } \\
\text { dificultades particulares }\end{array}$ \\
\hline & & $\begin{array}{l}\text { Colectiva } \\
\text { programada }\end{array}$ & $\begin{array}{l}\text { Los estudiantes } \\
\text { plantean sus dudas en } \\
\text { el aula o se resuelven } \\
\text { actividades de } \\
\text { evaluación previas }\end{array}$ & $\begin{array}{l}\text { Adquisición de } \\
\text { conocimientos y } \\
\text { competencias específicas y } \\
\text { transversales }\end{array}$ \\
\hline & & Entre iguales & $\begin{array}{l}\text { Los alumnos resuelven } \\
\text { casos prácticos } \\
\text { tutelados por un } \\
\text { alumno en el aula, con } \\
\text { la participación del } \\
\text { profesorado }\end{array}$ & $\begin{array}{l}\text { Adquisición de } \\
\text { conocimientos y } \\
\text { competencias } \\
\text { específicas y } \\
\text { transversales, } \\
\text { especialmente las } \\
\text { relacionadas con las } \\
\text { habilidades } \\
\text { interpersonales }\end{array}$ \\
\hline & Virtuales & Aula delegada & $\begin{array}{l}\text { Los estudiantes } \\
\text { plantean sus dudas al } \\
\text { tutor mediante correo }\end{array}$ & $\begin{array}{l}\text { Adquisición de } \\
\text { conocimientos y } \\
\text { competencias específicas y }\end{array}$ \\
\hline
\end{tabular}




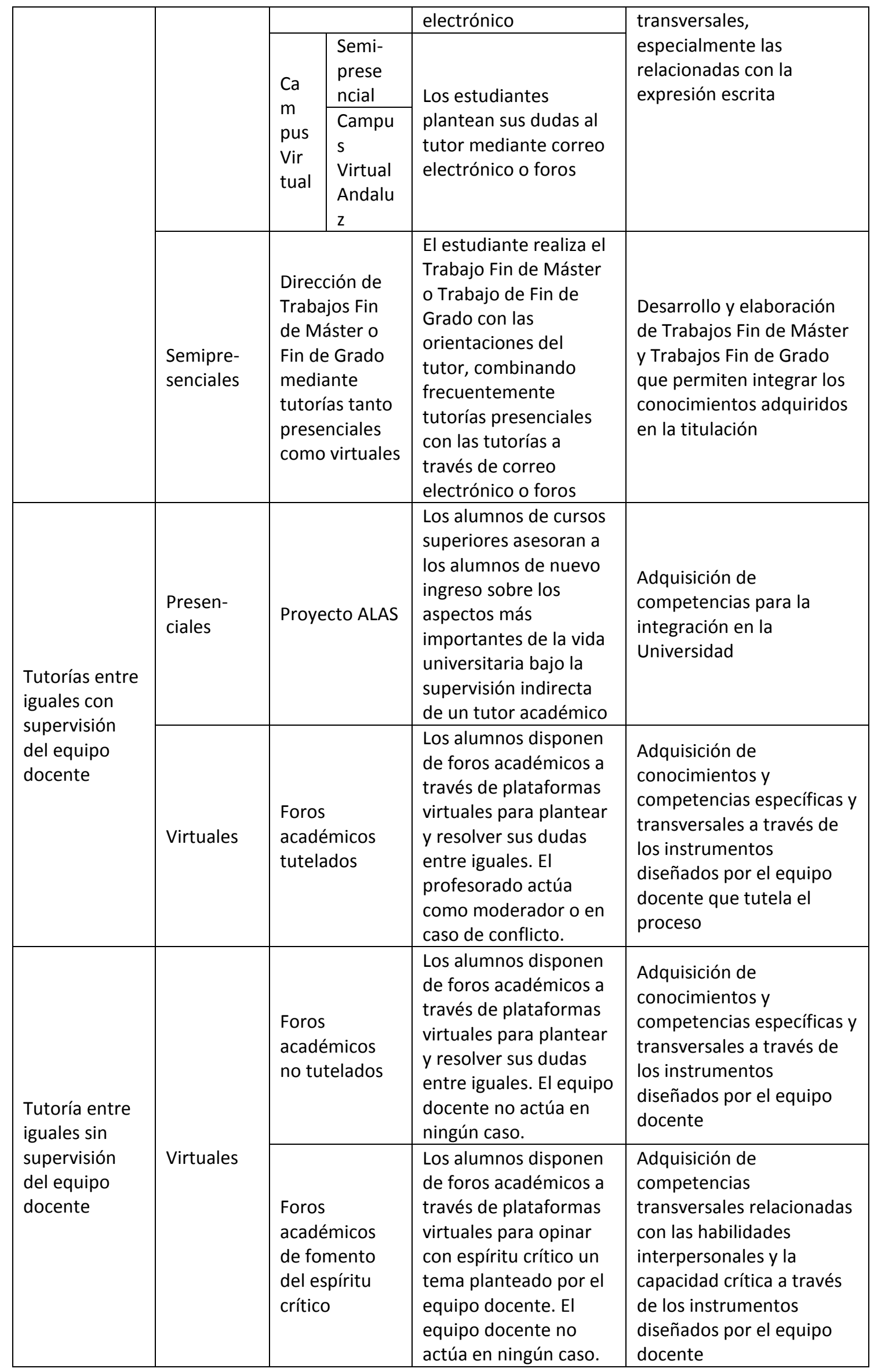

Tabla n. 1. Clasificación de las acciones tutoriales desarrolladas 


\section{Tutoría académica presencial individual}

Este tipo de tutoría coexiste con el resto de experiencias que planteamos seguidamente, no solo por su carácter legal, sino como pilar básico de la acción tutorial que permite al estudiante tanto solventar dudas de corte académico como cualquier otra dificultad que tenga el estudiante en el resto de acciones tutoriales desarrolladas.

\section{Tutoría académica presencial colectiva programada}

La tutoría colectiva programada se ha establecido, desde la implantación de las titulaciones de Grado, como un instrumento metodológico más, programado previamente junto al resto de sesiones teóricas y prácticas. Si bien se sigue planteando como un espacio en el aula donde los alumnos plantean las dudas que el profesor resuelve, para dotarlas de mayor contenido, habitualmente se establecen varias tutorías programadas a lo largo del semestre. A través de las mismas, el estudiante recibe un feedback personalizado sobre su progreso académico y se le comunica la puntuación que ha obtenido en cada una de las pruebas de evaluación que ha realizado, discutiéndose en el aula los errores cometidos y poniendo en común las mejores soluciones aportadas por los alumnos a los casos prácticos que han resuelto, de modo que todo el grupo avance en el aprendizaje de cara a la evaluación final de la materia.

La experiencia se valora muy positivamente tanto por los alumnos como por los profesores, ya que permite analizar el progreso de los alumnos conjuntamente. Si bien es imposible aislar el efecto del proceso de tutorización en la satisfacción del alumno, los resultados de los informes de satisfacción con la docencia universitaria correspondiente al curso $2011-2012^{2}$ de las asignaturas implicadas en la experiencia muestran una valoración global profesor/a - asignatura media de 4.3 sobre un máximo de 5 puntos.

\section{Tutoría académica presencial entre iguales}

Tras constatar que los alumnos tenían muchas reticencias a acudir a tutoría mientras que preferían solventar sus dudas consultando a sus compañeros se optó por implantar la tutoría entre iguales. No obstante, en estos procesos el aprendizaje no es supervisado por el profesorado que debe ser quien guíe el aprendizaje del alumno para que adquiera mecanismos de autoaprendizaje $y$, en muchas ocasiones, los conocimientos transmitidos no son correctos. Para intentar solventar este problema, se estableció en la asignatura Dirección Financiera (troncal, anual, de nueve créditos, de segundo curso de la Diplomatura en Ciencias Económicas y Empresariales) durante el curso académico 2009-2010, una serie de grupos donde un estudiante con mejor rendimiento era el encargado de enseñar al resto de miembros del grupo en las clases prácticas el modo de resolver un determinado supuesto que, previamente, había sido validado por el profesor. La experiencia fue muy positiva tanto para los alumnos

\footnotetext{
${ }^{2}$ Dicho informe es realizado por la Unidad de Evaluación y Calidad dependiente del Vicerrectorado de Prospectiva y Calidad de la Universidad de Cádiz.
} 
integrantes del grupo, que mejoraron su rendimiento académico como, especialmente, para los alumnos tutores, que fueron quienes obtuvieron mejores calificaciones finales. Los datos obtenidos del Sistema de Información de la Universidad de Cádiz correspondientes a las tasas medias de presentados, de éxito y de rendimiento de los tres cursos precedentes a la implantación de la experiencia se situaron respectivamente en el $57.9 \%, 76.2 \%$ y $42.9 \%$. Tras la implantación de la experiencia tutorial en el curso $2009 / 2010$ se situaron en el $71.5 \%, 88.9 \%$ y $63.5 \%$, respectivamente.

Adicionalmente, los alumnos valoraron especialmente cómo habían desarrollado otra serie de habilidades como la comunicación, la capacidad de resolución de problemas, el liderazgo y la autoestima. Durante el desarrollo de la experiencia, se constató como el uso de la tutoría tradicional aumentó, dado que los alumnos, en general, habían reducido sus reticencias para acceder al profesorado.

\section{Tutoría académica virtual en aulas delegadas}

La docencia en el aula delegada de Campus Bahía de Algeciras, que dista unos 120 kilómetros de la Facultad de Ciencias Económicas y Empresariales con sede en Cádiz, imponía grandes dificultades en la relación alumno-profesor. El no estar presencialmente en la Sede donde se impartía la docencia salvo el día en el que se impartían las clases presenciales impuso también una distancia geográfica con el alumnado que había que salvar. Para ello, tras los resultado académicos obtenido en el primer curso de implantación de la titulación (tasa de presentados: $35 \%$, tasa de éxito: 42.9\% y tasa de rendimiento: 15\%), se implantó en el curso académico 2005-2006 una experiencia, pionera en su día, de apoyo de la docencia a través de las Nuevas Tecnologías y el Campus Virtual (Giner y Muriel, 2010). En la asignatura Dirección Financiera (troncal, semestral, de nueve créditos, de cuarto curso de la Licenciatura en Administración y Dirección de Empresas), se facilitó al alumno, previamente a cada una de las sesiones docentes, todo el material a través del Campus Virtual (presentaciones correspondientes a las sesiones teóricas, casos prácticos, bibliografía, etc.). Adicionalmente, se implantó un sistema de tutorías electrónicas donde el alumno podía consultar con el profesorado cuantas dudas tuviese, y se fomentó su uso a través del envío sistemático de actividades evaluables (Araujo et al., 2005).

La puesta en marcha de esta experiencia fue valorada muy positivamente, mejorando las tasas de presentados, de éxito y de rendimiento sustancialmente, pasando a valores del $78.4 \%, 96.6 \%$ y $75.7 \%$ respectivamente, valores que se han mantenido en la misma línea durante los cursos académicos posteriores en los que se ha mantenido el sistema.

La experiencia se instrumentalizó a través del proyecto de innovación docente "Mejora de la enseñanza en aulas delegadas a través de plataformas virtuales" que recibió una mención honorífica por su calidad, otorgada por la Universidad de Cádiz durante el curso académico 2005/2006, sobre un total de 128 proyectos presentados. 


\section{Tutoría académica no presencial en el Campus Virtual}

Desde el curso académico 2003-04 se ha venido impartiendo con carácter semipresencial, en el Campus Virtual de la Universidad de Cádiz, las asignaturas Matemáticas Financieras, Matemáticas de las Operaciones Financieras y Operaciones Bancarias y Bursátiles; y desde el curso 2007-2008, en el Campus Virtual Andaluz la asignatura Operaciones Bancarias y Bursátiles (Toledano et al., 2010).

La puesta en funcionamiento de los Foros de Dudas ha permitido que los alumnos expusieran todo tipo de dudas referentes a las asignaturas, bien de contenidos o bien sobre la marcha de las mismas.

Inicialmente, el profesorado respondía personalmente a cada duda o bien a un conjunto de dudas, pero se observó que los alumnos amparándose en el "anonimato" de los mensajes en el foro se animaban a preguntar y participar, algo que no hacían en las horas presenciales que se impartían en las asignaturas semipresenciales.

En los siguientes cursos, y tras la positiva experiencia de las tutorías entre iguales desarrolladas en el aula y en los foros interactivos tutelados, se planteó que las tutorías fuesen entre iguales. Al incluir un incentivo en la calificación para aquellos que respondiesen correctamente a sus compañeros, la respuesta fue satisfactoria porque los alumnos empezaron a responder y con un nivel de precisión bastante elevado. El equipo docente diseñó todo el proceso para propiciar el autoaprendizaje del alumno, de modo que el profesorado estableció que no intervendría, en general, y sólo puntualizaría cuando las respuestas no fueran del todo correctas.

La validez de la experiencia tutorial es corroborada por el ascenso del $13.4 \%$ experimentado por la tasa media de éxito y del $7.8 \%$ correspondiente a la tasa media de rendimiento de las asignaturas semipresenciales comparando los resultados del curso en el que se puso en marcha la experiencia tutorial con los del curso anterior.

\section{Tutoría académica semipresencial para la dirección de TFM y TFG}

Se trata de una tutorización semipresencial orientada a la tutela efectiva del Trabajo Fin de Máster y, en el presente curso, la extensión a los Trabajos Fin de Grado que se imparten por primera vez en las titulaciones de Economía. Este tipo de tutorización se fundamenta en varias sesiones presenciales y se apoya constantemente en el uso de medios electrónicos para solventar, de una forma rápida, aquellas dudas que podrían impedir el avance del alumno en la elaboración de los trabajos.

No obstante, es necesario incidir en la riqueza de esta modalidad donde, en realidad, el proceso de tutorización con el alumno suele ir bastante más allá de la mera tutoría académica dado que tanto por la mayor intensidad de la relación con el alumno al que se le dirige el trabajo como al estar próximo el fin de sus estudios suelen realizarse tutorías de carácter profesional y personal, de orientación al alumno sobre posibles salidas laborales, idoneidad de las posibles decisiones a tomar sobre su futuro profesional y sus implicaciones para su vida personal. 


\section{Tutoría entre iguales presencial tutelada: el Proyecto ALAS}

El Proyecto ALAS (Alumnos Asesores), que inició su andadura en la Facultad de Ciencias Económicas y Empresariales en el curso académico 2001-2002, fue uno de los precursores de los actuales programas de acogida a estudiantes de nuevo ingreso implantados de manera sistemática por el EEES. Su objetivo, ratificado en la actualidad como una necesidad para adaptar al estudiante a la vida universitaria y mejorar las tasas de rendimiento y éxito académico a las que las memorias de los Grados se comprometen, se plantea con el objetivo de ofrecer ayuda a los alumnos de nuevo ingreso. Para ello, contamos con la colaboración de estudiantes de cursos superiores que prestan su apoyo, asesorando a sus compañeros a través de varias reuniones informativas presenciales que cuentan, siempre, con la supervisión de varios docentes que tutelan el proceso aunque, por supuesto, intervienen ante cualquier problema que presenten los alumnos.

Presenta un alto valor formativo para el alumno y claras ventajas en cuanto a economía de esfuerzos, así como la posibilidad de llegar, de forma viable, a un gran número de los estudiantes universitarios. Ello supone preparar en cada centro a un grupo de profesores que coordinan a los estudiantes tutores, seleccionar y preparar a los estudiantes tutores, reglamentar los derechos, obligaciones, formas de selección y créditos a conceder a los estudiantes tutores de cursos superiores que tuvieran a su cargo a los estudiantes de nuevo ingreso y dotar de una infraestructura para hacer posible el funcionamiento (despachos, lugares de encuentro, medios de comunicación,...).

\section{Tutoría entre iguales virtual tutelada a través de foros}

El conjunto de experiencias comentadas, vigentes durante varios cursos académicos, han sido la base sobre la que, tras la implantación de los Grados, se ha desarrollado el proceso de tutoría virtual que actualmente se lleva a cabo en varias asignaturas del Grado en Finanzas y Contabilidad: Introducción a las Finanzas Empresariales, Gestión Financiera, Valoración de las Operaciones Financieras y Planificación Financiera.

El bagaje previo sobre diversas modalidades de tutorías y el convencimiento de utilizar las nuevas tecnologías en el desarrollo cognitivo del alumno, nos han impulsado a emplearlas como metodología para favorecer el aprendizaje.

No obstante, la familiaridad del alumno con las nuevas tecnologías no está exenta de problemas cuando ésta se traslada al campo académico. En particular, en los últimos años se denota una menor riqueza en el lenguaje de los alumnos, múltiples errores gramaticales debido al uso incorrecto del lenguaje por traslación de las prácticas habituales en la escritura en redes sociales o mensajes de texto y, en general, una mayor dificultad de redacción de textos académicos o profesionales. Estos aspectos pretenden ser corregidos a través de los foros, enseñando al alumno como un mismo medio, un foro de Internet, debe utilizarse en un contexto académico con un lenguaje correcto, profesional, y que desarrolle en el alumno capacidades personales y profesionales que tendrá que aplicar en su futuro entorno laboral.

En este caso, no se puede analizar diferencias de rendimiento tras la experiencia tutorial dado que, desde su implantación, las tutorías forman parte de la programación 
integral de las asignaturas. No obstante, se observa como las tasa medias de algunos indicadores de las asignaturas implicadas desde el primer curso en que se impartieron hasta el curso 2011/2012 superan a los valores medios de dichos indicadores en la titulación del Grado en el que se insertan. Concretamente la tasa media de presentados está un $13.3 \%$ por encima de la de la tiutlación, la tasa media de éxito un $22 \%$ y la tasa media de rendimiento la supera un $26.8 \%$.

\section{Tutoría entre iguales virtual no tutelada a través de foros}

Tras las primeras experiencias, se hizo necesario dar un paso más para que el alumno desarrollase su proceso de aprendizaje en un contexto lo más próximo posible a la realidad, una realidad cambiante, con un gran cúmulo de información donde una de las capacidades básicas que un profesional debe tener es la selección de la información idónea.

Por ello, se planteó la posibilidad, tras el diseño del proceso y de los instrumentos por parte del equipo docente, de que los foros no fuesen tutelados en ningún caso, tanto para el desarrollo de la capacidad cognitiva del alumno como para fomentar la capacidad de resolver conflictos, valorándose especialmente la ayuda prestada a los compañeros resolviendo dudas, los consejos y los trabajos compartidos, etc., y, como lógica contrapartida, negativamente la falta de respeto y el uso incorrecto del lenguaje, si bien hay que señalar que este tipo de incidencias ha sido mínima.

La propia dinámica del foro, y como ha ocurrido en cursos anteriores, ha hecho que un buen número de alumnos participaran en el proceso de tutorización, respondiéndose entre ellos las dudas que iban surgiendo, sobre todo relacionadas con la resolución de las materias tratadas, bien de teoría o de ejercicios, problemas prácticos y demás actividades propuestas por el equipo docente, lo que ha permitido a los alumnos trabajar las competencias específica de las materias $y$, de igual modo, ha potenciado competencias transversales como liderazgo, autonomía, pensamiento crítico y capacidad de analizar información de fuentes diversas.

Desde el inicio, y como demuestra nuestra experiencia en el Campus Virtual, surgen, sin necesidad de nombrar un número de tutores que se encarguen de tutorizar cada uno de ellos a otro grupo de alumnos (alumnos tutorizados), lo que hemos llamado "tutores naturales" que son aquellos alumnos que van siguiendo la asignatura continuamente, que entienden perfectamente los conceptos, que realizan las actividades propuestas en sus fechas y que vienen a coincidir con aquellos que han obtenido buenas calificaciones en las pruebas realizadas. Estos tutores naturales, cuyo número depende del tamaño del grupo, son los que inicialmente animan y contagian al resto y consiguen que los compañeros se animen a seguir la asignatura.

Como se puede apreciar en la siguiente tabla, a modo de ejemplo ilustrativo se observa cómo en dos asignaturas del Grado en Finanzas y Contabilidad, las intervenciones del profesorado en la resolución de dudas es meramente testimonial comparada con la de los tutores naturales, objetivo que se pretendía con este tipo de tutorización: la gestión del autoaprendizaje por parte del alumno. 


\begin{tabular}{|c|c|c|c|c|c|c|c|}
\hline \multicolumn{8}{|c|}{ Introducción a las Finanzas Empresariales } \\
\hline \multirow[b]{2}{*}{ Curso } & \multirow[b]{2}{*}{ Alumnos } & \multirow[b]{2}{*}{$\begin{array}{l}\text { Dudas o } \\
\text { debates }\end{array}$} & \multirow[b]{2}{*}{ Respuestas } & \multicolumn{2}{|c|}{ Tutores Naturales } & \multicolumn{2}{|c|}{ Profesores } \\
\hline & & & & Número & $\begin{array}{l}\text { Interven- } \\
\text { ciones }\end{array}$ & Número & $\begin{array}{l}\text { Interven- } \\
\text { ciones }\end{array}$ \\
\hline 2009-10 & 107 & 354 & 1770 & 5 & 78 & 5 & 12 \\
\hline 2010-11 & 116 & 128 & 640 & 3 & 65 & 5 & 7 \\
\hline 2011-12 & 166 & 150 & 1050 & 4 & 72 & 2 & 11 \\
\hline 2012-13 & 144 & 145 & 725 & 2 & 55 & 2 & 8 \\
\hline \multicolumn{8}{|c|}{ Valoración de Operaciones Financieras } \\
\hline & & & & \multicolumn{2}{|c|}{ Tutores Naturales } & \multicolumn{2}{|c|}{ Profesores } \\
\hline Curso & Alumnos & $\begin{array}{l}\text { Dudas o } \\
\text { debates }\end{array}$ & Respuestas & Número & $\begin{array}{l}\text { Interven- } \\
\text { ciones }\end{array}$ & Número & $\begin{array}{l}\text { Interven- } \\
\text { ciones }\end{array}$ \\
\hline $2010-11$ & 84 & 95 & 380 & 2 & 24 & 2 & 3 \\
\hline 2011-12 & 76 & 74 & 296 & 1 & 25 & 2 & 2 \\
\hline
\end{tabular}

Tabla n. 2. Evolución de la acción tutorial en asignaturas del Grado en Finanzas y Contabilidad

En definitiva, las tutorías entre iguales presentan las siguientes características:

- Dado el alto grado de participación de los alumnos, el profesorado pasa a ser facilitador e impulsor del uso de los foros, supervisando las intervenciones.

- La inmediatez en obtener respuestas a las dudas hace que el alumno avance en el estudio más rápido.

- A los alumnos les supone un ahorro de tiempo y les evitan tener que desplazarse a la Facultad en horas en las que no suelen estar o bien no tienen que perder clases para asistir a tutoría, adaptando su proceso de aprendizaje a sus restricciones horarias.

- No obstante, tras vencer las reticencias a través de Internet, los alumnos se sienten más seguros para acudir a las tutorías presenciales con el equipo docente.

- Al tener que contestar a las dudas de los compañeros, mejora la capacidad de expresión escrita del alumno.

- El alumno aprende a discernir entre todas las informaciones que recibe, cuál es la adecuada para el problema que pretende resolver, dado que, a menudo, recibe varias respuestas diferentes de los compañeros ante la misma duda. 
- La relación así iniciada entre los alumnos permite mejorar las relaciones personales entre ellos.

- La motivación y el interés por las asignaturas han aumentado, como demuestran las elevadas tasas de presentados, ya que en caso contrario sin la colaboración de los compañeros tutores muchos no hubieran afrontado las asignaturas.

- La tutorización no solo se ha ejercido sobre los contenidos propios de la asignatura, sino que además se ha extendido al uso de las herramientas informáticas empleadas en el desarrollo de la misma (hoja de cálculo, presentaciones, editores de texto, etc.) dado que el equipo docente no ha impartido estos contenidos, solo les ha comunicado a los alumnos la obligación de manejarlos.

\section{Tutoría entre iguales virtuales tutelada a través de foros para el fomento del espíritu crítico}

No quisiéramos finalizar el trabajo sin avanzar lo que consideramos el siguiente paso en la evolución natural de la tutoría asociada a los nuevos canales de distribución de información: la tutoría como elemento de fomento del pensamiento crítico de los alumnos. En este sentido, gran parte de la información que recibimos en la actualidad no solo procede de los canales de prensa, radio o televisión tradicionales. Hay multitud de información a la que acceden los estudiantes a través de Internet o foros alternativos de información, especialmente aquella de contenidos más críticos o de carácter menos convencional. Con el objeto de facilitar a los estudiantes el conocimiento de dichos canales y el pensamiento crítico, en el presente curso estamos iniciando una nueva experiencia donde los alumnos han de localizar alguna evidencia documental relacionada con la materia que se oponga frontalmente a lo explicado en el aula, a fin de que sean capaces de valorarlo, discutirlo y, en su caso, opinar razonadamente sobre la veracidad o no de dichos documentos.

\section{Conclusiones}

La evolución del proceso de aprendizaje, dentro del EEES, no puede sustraerse ni del nuevo papel del estudiante que ha de ser capaz de gestionar su propio proceso de autoaprendizaje, ni de la evolución de las nuevas tecnologías, presentes de manera inherente en todo el desarrollo cognitivo tanto de alumnos como de profesores. Por ello, la evolución imparable del proceso de tutorización debe adaptarse a estos cambios y utilizarlos, del mejor modo posible, para adquirir competencias y capacidades no solo relacionadas con la materia en la que se insertan como una actividad metodológica más, y donde el rol de profesorado se centra en el diseño y supervisión de los medios para que el estudiante desarrolle la capacidad de autoaprendizaje.

En este sentido, las tecnologías de la información cobran una especial importancia, ya que no solo facilitan la comunicación entre tutor y alumno sin 
necesidad de estar en el mismo espacio y tiempo, sino que pueden aportar instrumentos de seguimiento y gestión. Adicionalmente, las ventajas que aporta el aprendizaje cooperativo, pueden aprovecharse para establecer tutoría entre iguales a través de foros interactivos, aunando ambos aspectos, de modo que se fomente en los estudiantes la curiosidad, la indagación, la iniciativa personal y colectiva y el contraste de opinión, además de permitir trabajar ciertas competencias genéricas como capacidad de liderazgo, la gestión de conflictos o la habilidad para analizar y buscar información proveniente de fuentes diversas, entre otras.

Como siguiente paso en la evolución tutorial, estamos convencidos del papel que Internet como medio de difusión de opinión puede aportar a los estudiantes para fomentar su espíritu crítico y el desarrollo de una opinión formada, al valorar y considerar distintas aportaciones que en el ámbito de la materia se generen a partir de diferentes enfoques o razonamientos.

Para finalizar, consideramos que el avance en el desarrollo de las metodologías que emplean la tutoría debe ir acompañado de la implicación de las autoridades académicas, para dar respuesta a los retos que implica su implantación: la formación de tutores, la participación de los alumnos, la planificación docente y, evidentemente, el reconocimiento de la tarea del tutor y la dotación de los recursos necesarios.

\section{Referencias bibliográficas}

Álvarez, M. (2008). La tutoría académica en el Espacio Europeo de la Educación Superior. Revista Interuniversitaria de Formación del Profesorado, 22 (1), 71-88.

Álvarez, P. (2005-2006). La tutoría y la orientación universitaria en la nueva coyuntura de la enseñanza superior: El programa 'Velero'. Contextos Educativos, 8-9, 281293.

Álvarez, P. R. y González, M. (2008). Análisis y valoración conceptual sobre las modalidades de tutoría universitaria en el Espacio Europeo de Educación Superior. Revista Interuniversitaria de Formación del Profesorado, 22 (1), 49-70.

Araujo, P., Giner, Y., Piñero, J. M. y Vélez, Mạ L. (2005). Mejora del acceso al material curricular a través de una plataforma virtual. En Unidad para la Calidad de las Universidades Andaluzas (UCUA) (Ed.), Proyectos de innovación docente en las universidades andaluzas. Memorias de los proyectos, curso 2003/2004 (pp. 5-31). Córdoba: Unidad para la Calidad de las Universidades Andaluzas (UCUA).

Barrón, Ma C. (2009). Docencia universitaria y competencias didácticas. Perfiles Educativos, XXXI (125), 76-87.

Capelo, Mạ D., Araujo, P., García-Borbolla, A., García, T., Giner, Y., Piñero, J. M., Ruiz, E. y Vélez, Má L. (2010). Adaptación de materiales y métodos docentes del área de Economía Financiera y Contabilidad a la docencia del Grado y Postgrado en Administración de Empresas. En Calzado, Mạ Y. y Martín, F. (Coord.), Reflexión sobre el Espacio Europeo de Educación Superior en la Facultad de Ciencias Económicas y Empresariales: hacia nuevos horizontes metodológicos (pp. 105111). Cádiz: Servicio de Publicaciones de la Universidad de Cádiz. 
Castaño, E., Blanco, A. y Asensio, E. (2012). Competencias para la tutoría: experiencias de formación con profesorado universitario. Revista de Docencia Universitaria, 10 (2), mayo-agosto, 193-210.

Gallego, S. (1997). Perfil del tutor universitario. En Apodaca, P., Arbizu, F., Lobato, C. y Olalde, C. (Comps.). Congreso Orientación Universitaria y Evaluación de la Calidad (pp. 67-74). Leioa, España.

García, N., Asensio, I., Carballo, R., García, M. y Guardia, S. (2005). La tutoría universitaria ante el proceso de armonización europea. Revista de Educación, $337,189-210$.

García, J. M. y Gálvez, M. (1996). Un modelo tutorial universitario. Revista Complutense de Educación, 7 (1), 51-66.

Giner, Y. y Muriel, Ma J. (2010). Mejora de la enseñanza en aulas delegadas a través de plataformas virtuales. En Calzado, Mạ Y. y Martín, F. (Coord.), Reflexión sobre el Espacio Europeo de Educación Superior en la Facultad de Ciencias Económicas y Empresariales: hacia nuevos horizontes metodológicos (pp. 43-47). Cádiz: Servicio de Publicaciones de la Universidad de Cádiz.

Lobato, C., del Castillo, L. y Arbizu, F. (2005). Las representaciones de la tutoría universitaria en profesores y estudiantes: Estudio de un caso. International Journal of Psychology and Psychological Therapy, 5 (2), 148-168.

Sancho, J. (2002). El sentido y las prácticas de las tutorías de asignaturas en la enseñanza universitaria. En Coriat, M. (Ed.), Jornadas sobre tutorías y orientación (17-36). Granada: Universidad de Granada.

Sogues, M., Gisbert, M. y Isus, S. (2007). E-tutoría: uso de las tecnologías de la información y comunicación para la tutoría académica universitaria. En García, J. y Seoane, A. M. (Coord.). Tutoría virtual y e-moderación en red. Monográfico en línea. Revista Electrónica Teoría de la Educación: Educación y Cultura en la Sociedad de la Información, 8 (2). Extraído el 3 de marzo de 2013, de http://www.usal.es/ teoriaeducacion/rev numero 08 02/n8 02 sogues gisber $\mathrm{t}$ isus.pdf

Sola, T. y Moreno, A. (2005). La acción tutorial en el contexto del Espacio Europeo de Educación Superior. Educación y Educadores, 8, 123-144.

Toledano, J., Cabrera, F., de Bernardo, M., Mora, M. y Sánchez, R. (2010). Virtualización de asignaturas. En Calzado, Ma Y. y Martín, F. (Coord.), Reflexión sobre el Espacio Europeo de Educación Superior en la Facultad de Ciencias Económicas y Empresariales: hacia nuevos horizontes metodológicos (pp. 37-41). Cádiz: Servicio de Publicaciones de la Universidad de Cádiz. 
Artículo concluido el 24 de julio de 2013

Cita del artículo:

Giner Manso, Y., Muriel de los Reyes, Ma J. y Toledano Redondo, F. J. (2013). De la tutoría presencial a la virtual: la evolución del proceso de tutorización. Revista de Docencia Universitaria. REDU. Número monográfico dedicado a Tutoría y Sistemas de orientación y apoyo a los estudiantes, Vol.11 (2) Mayo-Agosto. pp. 89-106. Recuperado el (fecha de consulta) en http://www.red-u.net/.

\section{Acerca de las autoras y autor}

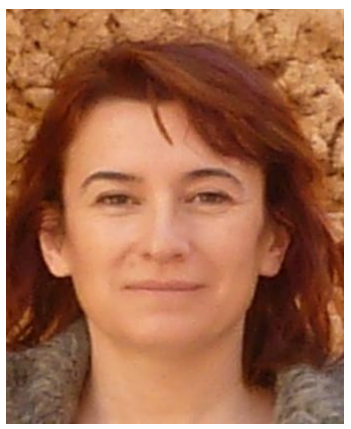

\section{Yolanda Giner Manso}

\section{Universidad de Cádiz}

Departamento de Economía Financiera y Contabilidad

Mail: yolanda.giner@uca.es

Doctora en Ciencias Económicas y Empresariales por la Universidad de Cádiz. Profesora Titular de Universidad en el Departamento de Economía Financiera y Contabilidad. Coordinadora del Grado en Finanzas y Contabilidad. Autora de publicaciones de carácter nacional e internacional relacionadas con las finanzas corporativas, la financiación de las PYMES y la innovación docente.

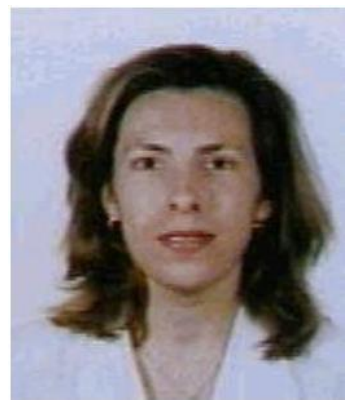

\section{María José Muriel de los Reyes}

\section{Universidad de Cádiz}

Departamento de Economía Financiera y Contabilidad

Mail: mariajose.muriel@uca.es

Doctora en Ciencias Económicas y Empresariales por la Universidad de Cádiz. Profesora Titular de Universidad en el Departamento de Economía Financiera y Contabilidad. Secretaria del Departamento de Economía Financiera y Contabilidad. Autora de publicaciones de carácter nacional e internacional relacionadas con las finanzas corporativas, la internacionalización de las empresas, el sistema financiero y la innovación docente. 


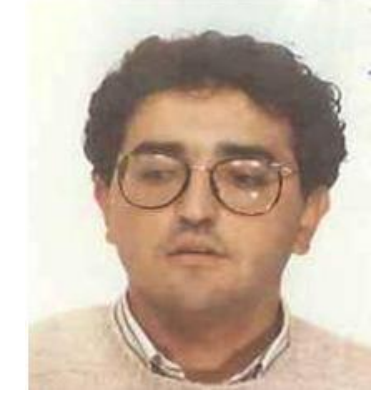

\section{F. Javier Toledano Redondo}

\section{Universidad de Cádiz}

Departamento de Economía Financiera y Contabilidad

Mail: javier.toledano@uca.es

Licenciado en Ciencias Económicas y Empresariales por la Universidad de Córdoba. Profesor Titular de Escuela Universitaria en el Departamento de Economía Financiera y Contabilidad. Es autor de publicaciones de carácter nacional e internacional relacionadas con las finanzas corporativas, la mortalidad empresarial y la innovación docente, con especial vinculación al Campus Virtual Andaluz. 